\title{
Optimization of the Digital Radio Mondiale Receiver Software Implementation to Support Digital Radio Mondiale Plus on DSP
}

\author{
Bharatha $\mathbf{H} \mathbf{P}^{1}$, P Nagaraju ${ }^{2}$ \\ Student, Telecommunication, R V College of Engineering,Bangalore, India ${ }^{1}$ \\ Associate Professor, Telecommunication, R V College of Engineering, Bangalore, India ${ }^{2}$
}

\begin{abstract}
Digital Radio Mondiale is a standard for digital radio broadcasting. To reduce number of cycles consumption, optimization is done to different modules of the current software implementation of the DRM receiver. Profiling is done to all modules. Cycle intensive modules are identified and optimized by using intrinsics. Correlation function achieved optimization with overall $\sim 69 \%$ reduction, resample module achieved optimization of $\sim 91.03 \%$ reduction, base band filter achieved $\sim 47 \%$ reduction and signal presence tacking module optimization resulted in $\sim 49.5 \%$ reduction. There is no degradation in the sensitivity of the receiver after optimization. Optimization has brought improvement in the processing time.
\end{abstract}

Keywords: Digital Radio Mondiale (DRM), Million Cycles per Second (MCPS), Intrinsic, Profiling.

\section{INTRODUCTION}

Digital Radio Mondiale (DRM) is a new digital radio A subset of MPEG xHE-AAC (Extended High-Efficiency broadcasting standard, to bring analog AM radio into Advanced Audio Coding), a subset of MPEG-4 AAC digital era. This was developed by DRM consortium (Advanced Audio Coding) and MPEG Surround (MPS) members and was approved by the European are used for source coding. Bit rate available for these Telecommunication Standard Institute (ETSI) in 2001. The coding are in the range from $8 \mathrm{kbit} / \mathrm{s}$ (half channels) to 20 perceived audio quality is much better and the reception is $\mathrm{kbit} / \mathrm{s}$ (standard channels) to up to $72 \mathrm{kbit} / \mathrm{s}$ (double more reliable. It also provides additional text and data channels) and bit rate for broadcasting channels above 30 services. It provides different transmission modes and $\mathrm{MHz}$ is in the range of $37 \mathrm{kbit} / \mathrm{s}$ to $186 \mathrm{kbit} / \mathrm{s}$.

different bandwidths to cope up with different channel conditions. Maximum bandwidth is $20 \mathrm{kHz}$ and no of DRM transmission super frame of $1200 \mathrm{~ms}$. It is divided OFDM carriers is less. These features motivate real time into three transmission frames of 400 ms. Super frame software implementation of a DRM receiver on a DSP. consists of three logical channels, Main Service Channel Through profiling cycle intensive modules are identified (MSC) which consists of payload, Fast Access Channel for the current software implementation and these are (FAC) which gives information about channel parameters optimized by loop unrolling and by using core specific and Service Description Channel (SDC) which gives intrinsic to reduce number of cycles consumption.

\section{SYSTEM OVERVIEW} information on how to decode the MSC. Before channel coding pseudo random binary code is used to scramble the bits and the unwanted regularity in the transmitted signal. Band of frequencies used for DRM broadcasting below 30 Multilevel coding is used in combination with QAM $\mathrm{MHz}$ are as follows, the low frequency band from 148.5 to modulation. Different QAM modulation for different $283.5 \mathrm{kHz}$ in region 1 , the medium frequency band from modes is given in the Table 1.

526.5 to $1606.5 \mathrm{kHz}$ in regions 1 and 3 and from 525 to $1705 \mathrm{kHz}$ in region 2 and the high frequency bands from 2.3 to $27 \mathrm{MHz}$ worldwide. Band of frequencies used for DRM+ broadcasting above $30 \mathrm{MHz}$ are as follows, analog television broadcasting (Band I) : $47 \mathrm{Mhz}$ to $68 \mathrm{MHz}$, OIRT FM Band : $65.8 \mathrm{MHz}$ to $74 \mathrm{MHz}$, Japanese FM Band : $76 \mathrm{MHz}$ to $90 \mathrm{MHz}, \mathrm{FM}$ Radio Broadcasting (Band II) : 87.5MHz to $107.9 \mathrm{MHz}$ and Digital Broadcasting (Band III) : $174 \mathrm{MHz}$ to $240 \mathrm{MHz}$.

Typically channel bandwidth for radio broadcasting below $30 \mathrm{MHz}$ is $9 / 10 \mathrm{kHz}$, it supports half channel bandwidth $4.5 / 5 \mathrm{kHz}$ to support simulcast with AM broadcasting and supports twice the nominal bandwidth i.e. $18 / 20 \mathrm{kHz}$ to provide larger transmission capacity. Channel width for radio broadcasting above $30 \mathrm{MHz}$ is $100 \mathrm{kHz}$.

Table 1.

Different modulation scheme for different modes and logical frames

\begin{tabular}{|c|c|c|}
\hline $\begin{array}{c}\text { Logical } \\
\text { Channels }\end{array}$ & $\begin{array}{c}\text { Mode A, B, } \\
\text { C and D }\end{array}$ & Mode E \\
\hline MSC & 64/16-QAM & 16/4-QAM \\
\hline FAC & 16/4-QAM & 4-QAM \\
\hline SDC & 4-QAM & 4-QAM \\
\hline
\end{tabular}

Pilot cells which are distributed in time and frequency are used for time, frequency and frame synchronization. These are also used for time tracking and channel estimation. 


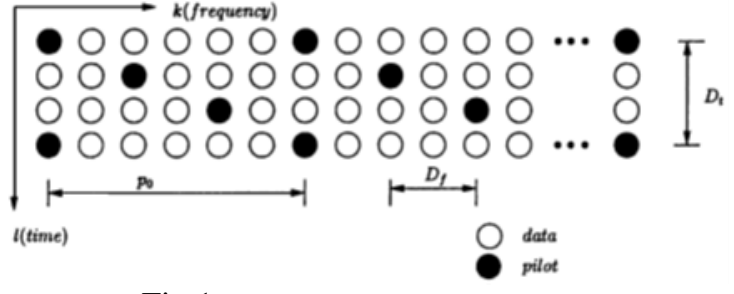

Fig 1: Pilot pattern form DRM mode B

\section{III.IMPLEMENTATION}

\section{A. Hardware and Software Requirement}

Hardware requirements are as follows, Computer, System on Chip (SoC), Test Transmitter, Coaxial cable, UART Cable, Speaker/Headphones and Power Supply to SoC 5v. Software requirements are as follows, GUI, Terminal v1.9 (debugging tool for serial communication), $\mathrm{C}$ code editor and $\mathrm{C}$ code compiler. Conceptual test setup is shown in the Fig 2.

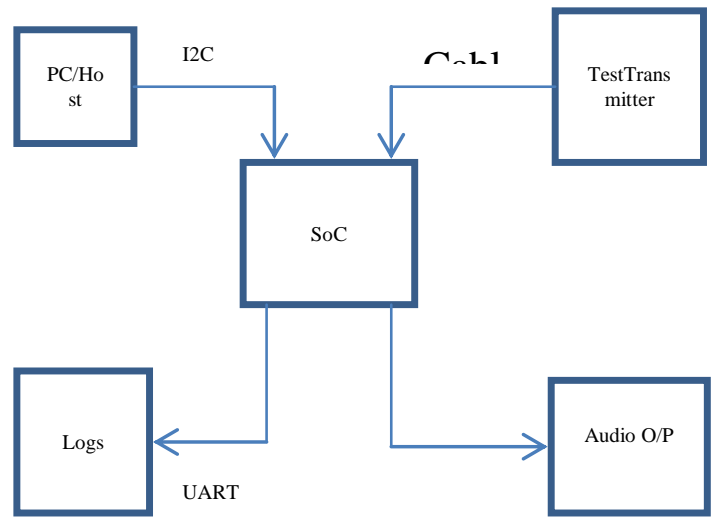

Fig 2: Conceptual Test Setup for DRM transmission and reception

\section{B. Profiling}

To know time and MCPS consumption from different modules, profiling is done on target broad and flow chart for profiling is as shown in the fig 3 .

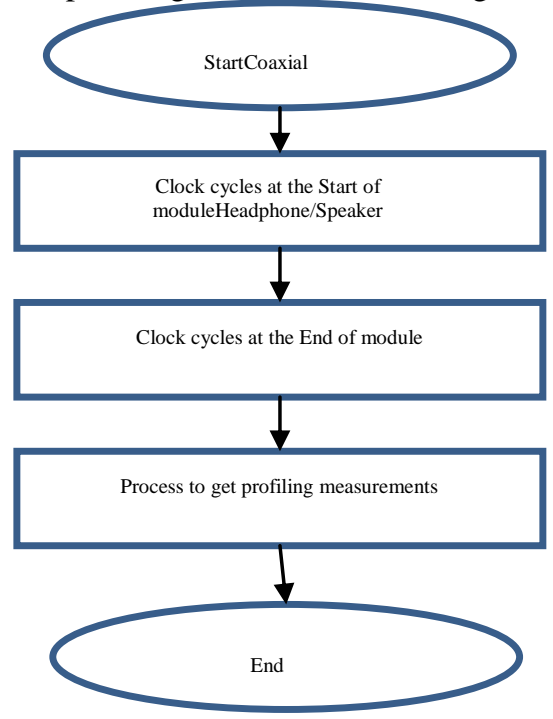

Fig 3: Flow chart for Profiling

To get profiling measurements test vector is modulated and transmitted from the test transmitter. Signals are transmitted through coaxial cables to the target board. Personal computer or host can be used to send commands to the target board through $\mathrm{I} 2 \mathrm{C}$ and SPI interface. Received signals are processed in the SoC and debugging messages are sent to the debugging terminal through UART cable and processed audio output is sent to speaker or to headphone as shown in the Figure 2.

Test vector specifications used for profiling are as follows, audio file format is .wav file, MSC uses 64-QAM modulation, bandwidth is $20 \mathrm{kHz}$, robustness mode is A, radio frequency output at the test transmitter is $95 \mathrm{dBuV}$ and target board clock frequency is $265 \mathrm{MHz}$.

In DRM receiver correlation module was consuming 6.54 MCPS, inputs to this module are real 16 bit filter coefficients and complex 16 bit data. Optimization is done 
as shown in the Figure 4 and there is $78.917 \%$ (i.e. 1.38

MCPS) reduction in the cycles consumption.

Resample is used to decimate input samples by 2 . Inputs to this function are 16 bit complex data, decimation factor is 2 and MCPS consumption before optimization is 23.87 and after optimization MCPS consumption is 2.14 . There is $91.03 \%$ reduction.

Base band filtering consumes 11.74 MCPS and inputs to this function are 16 bit complex filter coefficients and 16 bit complex data. After optimization it is taking 6.21 MCPS and there is $47.4 \%$ reduction in the cycle consumption.

Signal presence tracking is done to check whether signal is present by comparing averaged signal value with the threshold value. Input to this function is 16 bit complex data and there is $49.5 \%$ reduction in the MCPS consumption.

Optimised code is flashed onto the DRM receiver target board and Sensitivity test which measures the ability of the receiver to decode weak signals is done and compared with the minimum receiver requirements. There is no change in the sensitivity of the receiver with the optimized code.

\section{CONCLUSION}

Cycle intensive modules were successfully optimized by using core specific intrinsics. Code is successfully implemented on the target board and heard audio without any artifacts. Sensitivity testing is done for the same implementation, there is no degradation of the sensitivity and it matches with the minimum receiver requirements. Optimization have reduced number of arithmetic computations and it has brought improvement in processing time (speed up improvement) by reducing number of cycles consumption by different modules.

\section{REFERENCES}

[1] Frank Hofmann, Christian Hansen, and Wolfgang Schäfer, "Digital Radio Mondiale (DRM) Digital Sound Broadcasting in the AM Bands," IEEE Transactions on Broadcasting, Vol 49, NO 3, pp. 319 328 September 2003

[2] "Digital Radio Mondiale (DRM); System Specification," European Telecommunication Standards Institute (ETSI), ETSI ES 201980, 2014-01. 